Acta Crystallographica Section A

Foundations of Crystallography

ISSN 0108-7673

Received 20 September 2010

Accepted 29 October 2010

\section{On the allowed values for the triclinic unit-cell angles}

\author{
James Foadi $^{\mathrm{a} *}$ and Gwyndaf Evans ${ }^{\mathrm{b}}$
}

${ }^{\mathbf{a} M P L}$, Imperial College, London SW7 2AZ, England, and ${ }^{\mathbf{b}}$ Diamond Light Source Ltd, Harwell Science and Innovation Campus, Didcot, Oxfordshire OX11 0DE, England. Correspondence e-mail: j.foadi@imperial.ac.uk

This short article questions and investigates the possible range of values for the three angles of a unit cell in the triclinic system. Although no constraints are reported in manuals and tables for crystallography, the three angles are not really independent; the range of allowed values is calculated and presented in this paper.

\section{Introduction}

There is a broad, if not universal, acceptance by the community of crystallographers that cell parameters in the triclinic system can assume any arbitrary value. This is reported everywhere in textbooks, tables and papers on crystallography. It is certainly true that the $a, b$ and $c$ lengths of the cell can be given any value, and that they are quantities independent of each other. The same, though, cannot be stated for the angles; although each individual angle can assume any value between 0 and $180^{\circ}$, they are not independent of each other. This feature of the triclinic system is strongly related to the volume of the unit cell; wrong combinations of angles can result in the volume being zero or, even, an imaginary number.

In this short paper we will examine the whole issue quantitatively. It will be readily shown that the determinant appearing in the expression for the unit-cell volume is at the heart of the problem. A factorized version of that expression will enable us to single out the set of allowed values for the cell's angles. Finally, a simple geometric construction will help in clarifying the reason why the volume expression can have a null or imaginary value.

\section{The expression for the volume of a unit cell}

Throughout the text we will adopt a right-handed coordinate system; the main conclusions are identical if a left-handed system is chosen instead. The easiest way to derive the equation for the volume of a unit cell is through the triple product of its axes. This, in turn, is equivalent to the determinant of the matrix whose rows are the cell axis components (see, for instance, Giacovazzo et al., 2002, pp. 68-69, or Woolfson, 1997, pp. 61-62),

$$
V=\mathbf{a} \cdot[\mathbf{b} \times \mathbf{c}]=\operatorname{det}(A) \equiv\left|\begin{array}{lll}
a_{x} & a_{y} & a_{z} \\
b_{x} & b_{y} & b_{z} \\
c_{x} & c_{y} & c_{z}
\end{array}\right|
$$

When cell lengths and cell angles are preferred to Cartesian components, it is customary to square the volume. As the determinant of a transpose matrix is equivalent to the determinant of the matrix itself, we have

$$
\begin{aligned}
V^{2} & =[\operatorname{det}(A)]^{2}=\operatorname{det}(A) \operatorname{det}\left(A^{t}\right)=\operatorname{det}\left(A A^{t}\right) \\
& =\left|\begin{array}{lll}
\mathbf{a} \cdot \mathbf{a} & \mathbf{a} \cdot \mathbf{b} & \mathbf{a} \cdot \mathbf{c} \\
\mathbf{b} \cdot \mathbf{a} & \mathbf{b} \cdot \mathbf{b} & \mathbf{b} \cdot \mathbf{c} \\
\mathbf{c} \cdot \mathbf{a} & \mathbf{c} \cdot \mathbf{b} & \mathbf{c} \cdot \mathbf{c}
\end{array}\right|
\end{aligned}
$$

or

$$
V^{2}=a^{2} b^{2} c^{2}\left|\begin{array}{ccc}
1 & \cos \gamma & \cos \beta \\
\cos \gamma & 1 & \cos \alpha \\
\cos \beta & \cos \alpha & 1
\end{array}\right| \equiv a^{2} b^{2} c^{2} P
$$

Equation (1) for the square of the volume is interesting as it makes it immediately evident that cell lengths are independent of cell angles. From the same expression it also appears that determinant $P$ is of key importance in the calculation of the unit-cell volume. In the mathematical context of trihedral angles, its square root has been known as the generalized sine of the trihedral angle (Allendoerfer, 1965; Nahir, 1998). It is supposed to be a positive quantity, otherwise the volume will be zero or an imaginary number. Unfortunately, $P$ could assume null or negative values for certain combinations of angles. In fact, $P$ can assume any real value between -4 and 1 . Therefore, we will have to find the conditions on $\alpha, \beta$ and $\gamma$ that make $P$ positive. The analytic expression for $P$ is

$$
P=1-\cos ^{2} \alpha-\cos ^{2} \beta-\cos ^{2} \gamma+2 \cos \alpha \cos \beta \cos \gamma .
$$

Finding the conditions that make equation (2) positive can be quite complicated. It is preferable to use an equivalent factorized form (Allendoerfer, 1965; Sivardière, 1996),

$$
\begin{aligned}
P= & 4 \sin \left(\frac{\alpha+\beta+\gamma}{2}\right) \sin \left(\frac{\alpha+\beta-\gamma}{2}\right) \sin \left(\frac{\alpha+\gamma-\beta}{2}\right) \\
& \times \sin \left(\frac{\beta+\gamma-\alpha}{2}\right) .
\end{aligned}
$$

This equation has also been reported in International Tables for Crystallography (Donnay \& Donnay, 1959; Koch, 2004), and it is frequently used in computational programs for crystallography. A demonstration of how equation (3) can be derived from equation (2) is shown in Appendix $A$. 


\section{Determination of the allowed range for the unit-cell angles}

It is straightforward to find out when $P$ is positive if equation (3) is used. Simply, the product of its factors will have to be positive. This can happen if all factors have a positive sign, or if all have a negative sign, or if two factors are positive and two are negative. We can limit $\alpha, \beta$ and $\gamma$ to values in the range $[0,180]$, as numbers higher than these correspond to a specular image of the same unit cell, better described with a left-handed coordinate system. We will, therefore, be looking to a region where $P$ is positive, which is contained in the cube with a vertex at $(0,0,0)$ and with side equal to 180 . Each sine factor in the expression will be positive or negative on either of the sides of a specific plane. For example, $\sin [(\alpha+\beta+\gamma) / 2]$ will be positive above the plane of equation

$$
\alpha+\beta+\gamma=0
$$

and below the plane of equation

$$
\alpha+\beta+\gamma-360=0
$$

This intersects the cube in a closed, convex region; in this part of the cube all combinations of the three angles will give a positive value for the sine, in the complementary region of the cube to this the sine will be negative. By considering all possible combinations of signs for the sines and by inspecting, for each combination, which region of the cube is positive and which is negative, we are able to determine a convex region within which $P$ is positive. This is the interior of the tetrahedron depicted in Fig. 1, whose vertices have coordinates $A \equiv(0,0,0), B \equiv(180,0,180), C \equiv(0,180,180)$ and $D \equiv(180,180,0)$.

As the volume of the tetrahedron is one third of the volume of the cube, there are, in fact, going to be two times more combinations of $(\alpha, \beta, \gamma)$ giving a null or imaginary volume than those giving a positive, admissible volume. From a purely quantitative point of view, the three angles $\alpha, \beta$ and $\gamma$ will be valid unit-cell angles if they satisfy at the same time the following four inequalities:

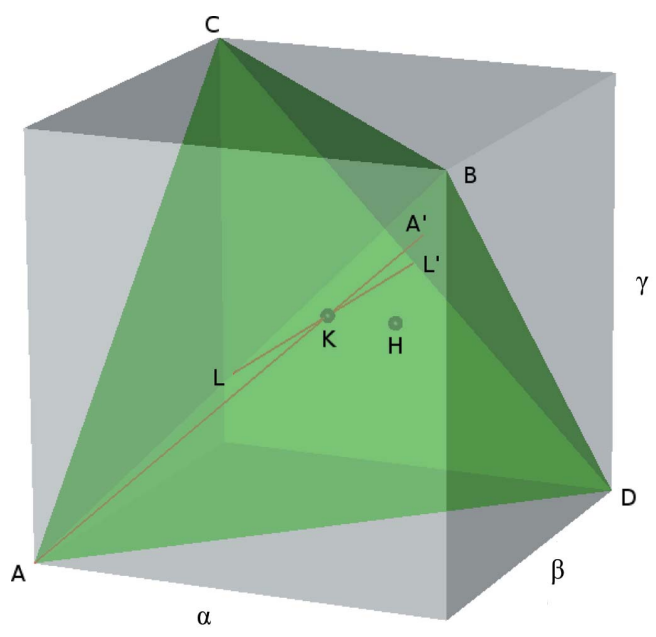

Figure 1

Each point inside this tetrahedron has coordinates $(\alpha, \beta, \gamma)$ which constitute allowed values for the unit cell of a triclinic system. The extra lines and points depicted inside the tetrahedron correspond to the angles of crystal systems different from the triclinic (see text).

$$
\left\{\begin{array}{l}
0<\alpha+\beta+\gamma<360 \\
0<\alpha+\beta-\gamma<360 \\
0<\alpha-\beta+\gamma<360 \\
0<-\alpha+\beta+\gamma<360 .
\end{array}\right.
$$

For example, a unit cell with angles 60, 60 and 130 cannot exist, despite the fact that they numerically appear perfectly sensible values for a triclinic cell. Fig. 1 also shows angle combinations corresponding to those systems different from the triclinic. Point $K \equiv(90,90,90)$ corresponds to the orthorhombic, tetragonal and cubic systems, point $H \equiv(90,90,120)$ to the hexagonal one. The straight line going from $L \equiv(90,0,90)$ to $L^{\prime} \equiv(90,180,90)$ corresponds to the monoclinic system, where the $\beta$ angle can assume any value in the range $(0,180)$. Finally, the straight line going from $A \equiv(0,0,0)$ to $A^{\prime} \equiv(120,120,120)$ corresponds to the rhombohedral system, where all three cell angles are equal to a single value between 0 and 120 . It is immediately obvious that all these points lie inside the tetrahedron, as it should be.

\section{A geometrically intuitive explanation for null and imaginary volumes}

In the previous section, quantitative arguments for unit-cell angle constraints have been presented. It would be desirable to find a qualitative or intuitive explanation of why there are constraints in the first place. The following simple geometric construction should provide the reader with such an explanation (Fig. 2).

Let $\gamma$ be the angle between axes $\mathbf{a}$ and $\mathbf{b}$ of the cell. The third axis, $\mathbf{c}$, will form an angle $\alpha$ with the $\mathbf{b}$ axis and an angle $\beta$ with the a axis. This means that $\mathbf{c}$ must lie at the same time on the surface of two cones with vertices at the origin of the unit cell. The first has its axis coincident with a and half-angle equal to $\beta$, while the second has its axis coincident with $\mathbf{b}$ and half-angle equal to $\alpha$. For certain combinations of the three angles the two cones will intersect in two straight lines starting from the origin and diverging specularly from the plane

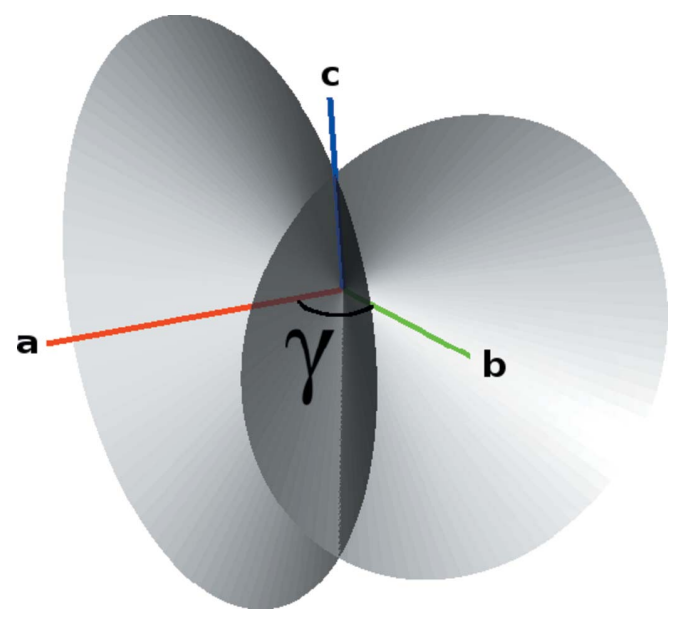

Figure 2

In this geometric construction, two of the three unit-cell axes, $\mathbf{a}$ and $\mathbf{b}$, are the central axes for two cones of half-angles $\beta$ and $\alpha$, respectively. When the cones intersect in two straight lines, then two specularly equivalent unit cells are constructed, and the third unit-cell axis, c, coincides with any of the two intersections. If the cones' apertures are progressively made to shrink, the two intersections will get closer and closer, until they coincide with a single straight line lying on the a-b plane; at this point the unit cell will have collapsed to a plane, and its volume is null. Further shrinking will have the effect of making even that single intersection disappear; in this case we have no unit cell and, consequently, its volume is an imaginary number. 
formed by $\mathbf{a}$ and $\mathbf{b}$; in this case $\mathbf{c}$ coincides with any of the two straight lines, and two specular unit cells will be constructed. When the cones' half-angles shrink beyond a certain value, though, the two straight lines will converge towards a single line on the $\mathbf{a}-\mathbf{b}$ plane, and further shrinking will result in no intersection at all. In the first case the unitcell volume will become zero, and the cell will degenerate into a parallelogram; in the second case the volume will be imaginary and no unit cell can be built.

\section{Discussion}

A unit cell describing a crystallographic structure must, quite obviously, have a finite, not imaginary, volume. In this sense, Nature is never going to be fooled by wrong combinations of angles, as they only occur for null or imaginary volumes. Constraints [equation (4)] are only useful in the context of a mathematical theory or a computer algorithm. The authors were led to the investigations underlying this paper when they tested their $c R y$ package for statistical applications in crystallography (Waterman et al., 2011) with unit-cell angles 60, 20, 80 (corresponding to a null volume) and found inconsistent, infinite results. Their software now includes conditions [equation (4)] to check against wrongly designed cells.

\section{APPENDIX $A$}

\section{A factorized expression for the determinant $\boldsymbol{P}$}

Using the following trigonometric identity

$$
\cos ^{2} \alpha=[1+\cos (2 \alpha)] / 2,
$$

let us replace all squared cosines in equation (2). This yields

$$
P=-1 / 2-[\cos (2 \alpha)+\cos (2 \beta)+\cos (2 \gamma)] / 2+2 \cos \alpha \cos \beta \cos \gamma .
$$

With another trigonometric identity, turning a sum into a product,

$$
\cos \alpha+\cos \beta=2 \cos [(\alpha+\beta) / 2] \cos [(\alpha-\beta) / 2],
$$

equation (6) is readily transformed into

$$
P=-[1-\cos (2 \gamma)] / 2-\cos (\alpha+\beta) \cos (\alpha-\beta)+2 \cos \alpha \cos \beta \cos \gamma
$$

and, using again the identity [equation (5)],

$$
\begin{aligned}
P= & -\cos ^{2} \gamma-\cos (\alpha+\beta) \cos (\alpha-\beta)+\cos (\alpha+\beta) \cos \gamma \\
& +\cos (\alpha-\beta) \cos \gamma .
\end{aligned}
$$

This last expression can easily be factorized as follows

$$
P=[\cos \gamma-\cos (\alpha-\beta)][\cos (\alpha+\beta)-\cos \gamma] .
$$

Equation (7) can be turned into the final factorized formula [equation (3)] once the trigonometric identity

$$
\cos \alpha-\cos \beta=-2 \sin [(\alpha+\beta) / 2] \sin [(\alpha-\beta) / 2]
$$

is applied to each of its factors.

The authors would like to thank one of the referees for the suggestion to stress the right-handedness of the system of axis chosen in the text. This work was supported by grant No. WT089809MA from the Wellcome Trust.

\section{References}

Allendoerfer, C. B. (1965). Math. Mag. 38, 253-259.

Donnay, J. D. H. \& Donnay, G. (1959). International Tables for X-ray Crystallography, Vol. II. Birmingham: Kynoch Press.

Giacovazzo, C., Monaco, H. L., Artioli, G., Viterbo, D., Ferraris, G., Gilli, G., Zanotti, G. \& Catti, M. (2002). Fundamentals of Crystallography, 2nd ed. Oxford University Press.

Koch, E. (2004). International Tables for Crystallography, Vol. C. Dordrecht: Kluwer Academic Publishers.

Nahir, Y. (1998). Int. J. Math. Educ. Sci. Technol. 29, 65-82.

Sivardière, J. (1996). Acta Cryst. A52, 955-956.

Waterman, D., Evans, G. \& Foadi, J. (2011). In preparation.

Woolfson, M. M. (1997). An Introduction to X-ray Crystallography, 2nd ed. Cambridge University Press. 